\title{
Methods for Detection of Astrophysical Tau Neutrinos in IceCube
}

\section{Tomasz Palczewski ${ }^{*}$}

Department of Physics and Astronomy, The University of Alabama, Tuscaloosa, AL, 35487

E-mail: tomas.j.palczewski@ua.edu

The IceCube Neutrino Observatory has reported the discovery of a diffuse flux of TeV-PeV astrophysical neutrinos at $5.7 \sigma$ significance from an all-flavor search. Neutrinos are expected to be produced in hadronic interaction processes at the sources of high-energy cosmic rays. Possible astrophysical sources include the gamma-ray bursts (GRBs), microquasars, supernovae, and active galactic nuclei (AGNs). The expectation for the neutrino flavor ratio at the source is 1: 2: $0\left(v_{e}: v_{\mu}: v_{\tau}\right)$. The flavor ratio of astrophysical neutrinos measured by ground-based detectors is modified due to neutrino oscillations averaged over astronomical distances. In particular, tau neutrinos should appear in the astrophysical neutrino flux and be detectable at Earth. Thus, tau neutrino searches are crucial to better determine the flavor composition. Methods to identify high-energy tau neutrino interactions in IceCube, a cubic-kilometer neutrino detector deployed in the glacial ice at the geographical South Pole, are described. An algorithm is presented for detecting a double pulse signature in the IceCube sensor signal, which can be an indication of the tau neutrino interaction and subsequent decay of the tau lepton inside the detector. Recent results for astrophysical tau neutrinos with three years of IceCube data is shown. Future prospects for tau neutrino detection in IceCube are discussed.

The European Physical Society Conference on High Energy Physics

22-29 July 2015

Vienna, Austria

\footnotetext{
* Speaker.

${ }^{\dagger}$ for the IceCube Collaboration (https://icecube.wisc.edu/collaboration/authors)
} 


\section{Introduction}

Recently, the IceCube collaboration announced the discovery of a high-energy diffuse flux of astrophysical neutrinos [1,2]. The flavor composition of the detected flux is consistent with equal fractions of all neutrino flavors [3, 4]. The identification of a high-energy tau neutrino would be an additional evidence confirming this result. The source of this flux is as yet unknown. Candidate neutrino sources include active galactic nuclei, gamma-ray bursts, microquasars, and supernova remnants $[5,6]$. Tau neutrinos are not produced in standard cosmic-ray atmospheric interactions and are expected at negligible levels in the prompt atmospheric neutrino flux [7]. In addition, oscillations of atmospheric $v_{e}$ and $v_{\mu}$ into $v_{\tau}$ are very limited at $\mathrm{TeV}$ and $\mathrm{PeV}$ energies and a travel distance comparable to the Earth diameter. In contrast, high energy $v_{e}$ and $v_{\mu}$ neutrinos of astrophysical origin can oscillate over cosmic distances to produce a measurable number of $v_{\tau} \mathrm{s}$ at the detector. Assuming standard 3-flavor oscillations and 1:2:0 $\left(v_{e}: v_{\mu}: v_{\tau}\right)$ flux at the source, it is expected that flavor ratio at the detector will be close to $1: 1: 1$ [8]. Therefore, the identification of astrophysical $v_{\tau}$ would be an independent confirmation of observed TeV-PeV energy astrophysical neutrino flux and it would be an important contribution to a measurement of the flavor composition.

\section{IceCube Detector}

IceCube is the world's largest neutrino detector, located near the geographic South Pole, instrumenting more than a cubic-kilometer of glacial ice. The detector consists of 86 cables ("strings"), each instrumented with 60 Digital Optical Modules (DOMs) deployed between depths of 1450 $\mathrm{m}$ and $2450 \mathrm{~m}$. [9] The DOM consists of a 10-inch photomultiplier tube (PMT), calibration light sources, and digitizing electronics. [10]. The digitized PMT signal is called a waveform.

\section{Detection Principle}

Neutrinos are detected in IceCube by observing the Cherenkov light produced by charged particles created when neutrinos interact in the ice. The neutrino interaction events can be generally classified into three different groups, based on the characteristic patterns of the Cherenkov light that provides information about the energy, direction, and flavor of the parent neutrino.

- Track-like events (see Fig. 1, left panel) have a good angular resolution (typically $<1^{\circ}$ ) but limited energy resolution when not fully contained in the detector volume. These events are produced mainly from the $v_{\mu} \mathrm{CC}$ interactions.

- Cascade-like events (see Fig. 1, middle panel) have a good energy resolution $(\sim 10 \%$ at high energies) but limited angular resolution (typically $>10^{\circ}$ ). They are described by a distinct spherical topology in the detector volume. This pattern results from $v_{e}, v_{\mu}, v_{\tau} \mathrm{NC}$ and $v_{e}, v_{\tau}$ CC interactions.

- Composite events (see Fig. 1, right panel) are a mixture of track-like and cascade-like events or multiple cascade events. This pattern has been produced by $v_{\mu} \mathrm{CC}$ interactions and is expected for $v_{\tau}$ CC interactions (not yet observed). 

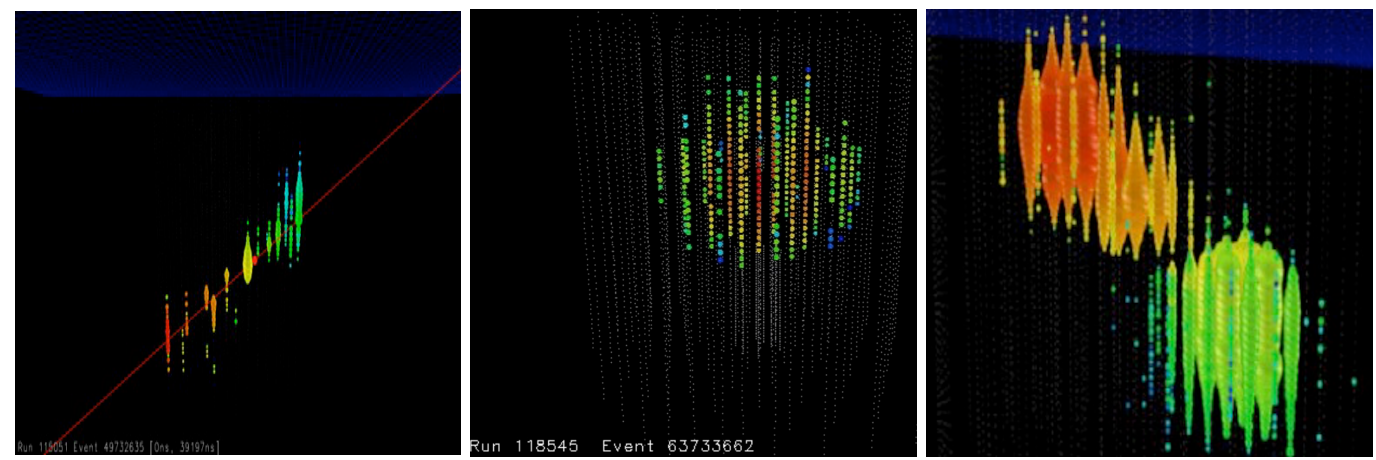

Figure 1: Neutrino interaction event topologies in IceCube: track-like event topology (left), cascade-like event topology (middle), and composite event topology (right). The size of the individual DOMs indicates the relative measured number of photoelectrons while the color represents the photon arrival times with red being early and blue late.

\section{Methods to Identify High-Energy Tau Neutrino Interactions}

Due to a large number of tau lepton decay modes and its long decay length at ultrahigh ( $\mathrm{TeV}$ and $\mathrm{PeV}$ ) energies, $v_{\tau}$ interactions have a rich set of possible signatures in the IceCube detector. The tau decay length is about $50 \mathrm{~m}$ per PeV. Tau leptons with decay lengths less than $1 \mathrm{~km}$ can be fully contained in the detector volume. Therefore, we can classify $v_{\tau}$ events into several categories based on the information about tau production and decay vertices:

- Double Bang: when $v_{\tau}$ interaction occurs inside the detector (visible hadronic shower) and tau decays in the modes producing a second cascade $\left(\tau \rightarrow e v_{e} v_{\tau}\right.$ and $\tau \rightarrow$ hadrons $v_{\tau}$, total branching ratio $(B R) \approx 82 \%$ ) inside the detector [8]. The tau lepton connecting the two showers will also emit Cherenkov light.

- Double Pulse: when two showers of a Double Bang event cannot be resolved as two separated cascades, there is an energy region where one or more DOMs near the showers will see double pulse waveforms.

- Partially contained Double Bang: two types of events contribute to this category:

- tau lepton from $v_{\tau}$ interaction is produced outside the detector and the initial hadronic shower is not visible but the tau enters the fiducial volume and decays to produce a shower $(\mathrm{BR} \approx 82 \%)$.

- tau lepton from $v_{\tau}$ interaction is produced inside the detector and then decays undetectably outside the fiducial volume (no BR factor).

- muonic tau decay: a tau from the $v_{\tau}$ interaction decays in the muonic tau decay channel (BR $\approx 18 \%$ ). One can distinguish two types of events in this class:

- tau lepton from $v_{\tau}$ interaction is produced outside the detector but it decays in muonic channel inside the detector. 
- tau from the $v_{\tau}$ interaction is produced and decays in the muonic tau decay channel inside the detector.

\section{Double Pulse Analysis}

The goal of double pulse analysis is to use individual DOMs to detect double bangs which are not well resolved into separate cascades [11]. Such events may produce a double pulse waveform in the DOMs. An example of simulated double pulse waveform is shown in Fig. 2. The same signature can be mimic by several types of background events:

- high-energy atmospheric muons or muon bundles passing through the detector

- $v_{\mu} \mathrm{CC}$ interactions with succeeding energetic muons

- scattered photons from single cascade events from $v_{e}, v_{\mu}, v_{\tau} \mathrm{NC}$ and $v_{e} \mathrm{CC}$ interactions that arrive late.

The double pulse algorithm to determine rising and trailing edges has been developed based on the first derivatives of a waveform. This search uses 914.1 days of data from the completed IceCube detector between May 13, 2011 and May 6, 2014. Event selection criteria, carried out at three cut levels (see [11] for more information), were developed using simulation and $10 \%$ of the data. The initial event selection was based on the Extremely High Energy (EHE) filter to select only high energy $v_{\tau}$ interactions where double pulses signature can be resolved. An additional charge cut on the number of observed photoelectrons $\left(\log _{10}(\mathrm{QTot})>3.3\right)$ was applied and waveforms were examined for double pulse characteristics (level 4). At the next level (level 5), track-like double pulse events, that are predominantly due to atmospheric muons, were removed using a cut on the reduced log likelihood ratio between a hypothesis of an infinite track and a hypothesis of a pointlike cascade. This cut has eliminated most down-going energetic muons and muon bundles. To further reduce down going muons, the first hit in the event was required to be below the top 40 meters of the instrumented volume. At the end (level 6) a containment cut was applied to eliminate cosmic ray induced muons which pass near the edges of the detector and appear as cascade-like events. Figure 2 (left) summarizes the passing rate of signal and background events at each cut level. Table 1 shows predicted event rates from all sources at the final cut level. No candidate

\begin{tabular}{|c|c|}
\hline Data samples & Events in 914.1 days (final cut) \\
\hline Astrophysical $v_{\tau} \mathrm{CC}$ & $(5.4 \pm 0.1) * 10^{-1}$ \\
\hline Astrophysical $v_{\mu} \mathrm{CC}$ & $(1.8 \pm 0.1) * 10^{-1}$ \\
\hline Astrophysical $v_{e}$ & $(6.0 \pm 1.7) * 10^{-2}$ \\
\hline Atmospheric $v$ & $(3.2 \pm 1.4) * 10^{-2}$ \\
\hline Atmospheric muons & $(7.5 \pm 5.8) * 10^{-2}$ \\
\hline
\end{tabular}

Table 1: Predicted event rates from all sources at the final cut level. Errors are statistical only. The assumed astrophysical flux is $E^{2} \Phi_{v}=1.0 * 10^{-8} \mathrm{GeV} \mathrm{cm}^{-2} \mathrm{sr}^{-1} \mathrm{~s}^{-1}$

events were observed in three years of IceCube data. An integrated astrophysical $v_{\tau}$ flux upper limit of $5.1 * 10^{-8} \mathrm{GeV} \mathrm{cm}^{-2} \mathrm{sr}^{-1} \mathrm{~s}^{-1}$ was derived assuming an astrophysical neutrino spectrum 

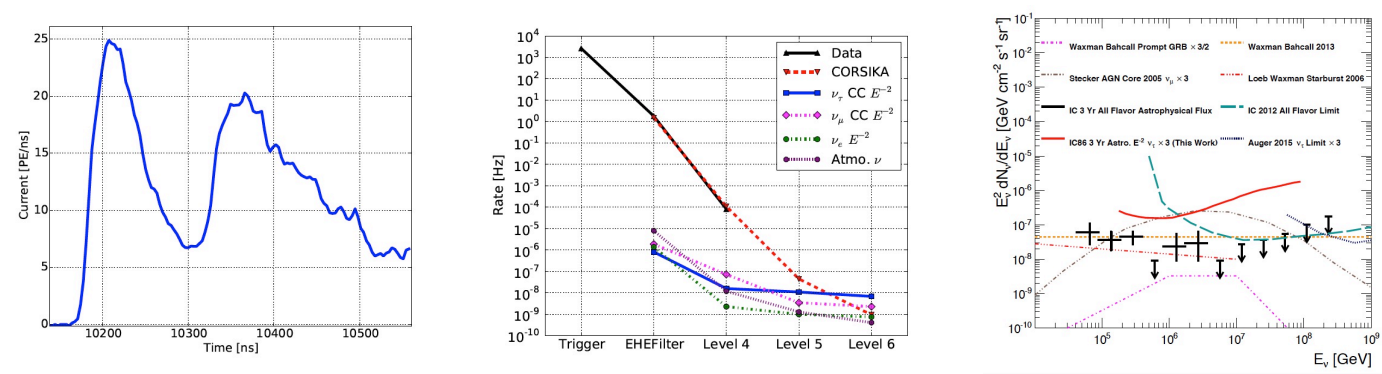

Figure 2: A simulated double pulse waveform obtained in one DOM from a $v_{\tau} \mathrm{CC}$ event (left). Passing rate for signal and backgrounds (middle). Neutrino flux upper limits and models as a function of the primary neutrino energy (right). The red curve is the differential upper limit derived from double pulse analysis (statistical and systematic errors are shown). The black error bars correspond to all flavor astrophysical neutrino flux observed by IceCube. [2].

with a spectral index of -2 . The differential upper limit on $v_{\tau}$ flux in the energy region of $214 \mathrm{TeV}$ to $72 \mathrm{PeV}$ was also obtained (see Fig. 2 (right)).

\section{Double Bang Analysis}

The double bang analysis looks for well separated double bangs [12]. The event selection starts with the EHE filter to select only high energy $v_{\tau}$ interactions. Fig. 3 presents the effective areas after the initial EHE cut for a few $v_{\tau}$ double bang event samples with different minimum separations between the two cascades. The energy threshold for the $v_{\tau}$ double bang identification

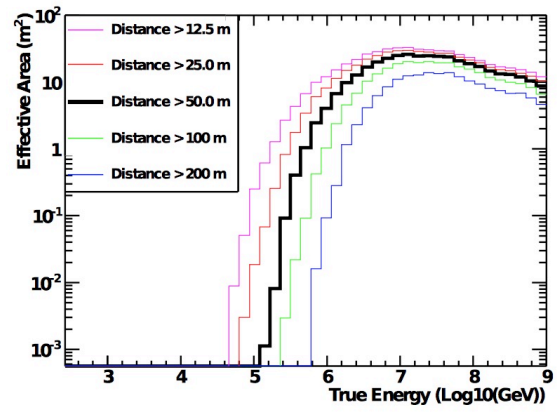

Figure 3: Effective areas after the initial EHE filter cut for $v_{\tau}$ obtained by selecting contained double bang events with different minimum separations between the two cascades. No reconstruction errors are included.

increases with distance between the two cascades. At the next cut level (level 4), a stronger cut on the number of observed photoelectrons is applied (total charge deposited in the event $>3100 \mathrm{PE}$ ). Then a Boosted Decision Tree (BDT) is used to further suppress background (level 5). The BDT was trained using well contained simulated $v_{\tau}$ double bang events, with a minimum separation between cascades of $50 \mathrm{~m}$, as a signal and simulated cosmic ray muons as a background. At the next level (level 6), a detailed reconstruction based on the assumptions of double bang topology 
was performed. To clean up the sample, several additional selection criteria were applied: The likelihood values obtained from the reconstruction cannot be too small, both cascades need to be reconstructed in or near the detector, the reconstructed separation between cascades cannot be smaller than $20 \mathrm{~m}$, and the reconstructed energy asymmetry of cascades ( $\left[E_{1}-E_{2}\right] /\left[E_{1}+E_{2}\right]$, where $E_{1}$ and $E_{2}$ are energies of the first and second cascade, respectively) has to be between 0.999 and 0.9 . The preliminary event rates of the signal and different backgrounds at the final level are shown in Table 2. The biggest remaining background comes from atmospheric muons. Work

\begin{tabular}{|c|c|}
\hline Data samples & Events in 1 year (final cut) \\
\hline Double Bang & $(4.93 \pm 0.04) * 10^{-1}$ \\
\hline Atmospheric muons & $(9.5 \pm 1.8)$ \\
\hline Astrophysical $v_{e}$ & $(8.2 \pm 0.3) * 10^{-1}$ \\
\hline Astrophysical $v_{\mu}$ & $(8.9 \pm 0.2) * 10^{-1}$ \\
\hline Atmospheric $v_{e}$ & $(4.4 \pm 0.2) * 10^{-2}$ \\
\hline Atmospheric $v_{\mu}$ & $(9.3 \pm 0.2) * 10^{-2}$ \\
\hline
\end{tabular}

Table 2: Preliminary remaining event rates of signal and background contributions at the final level of double bang analysis.

is underway to further reduce this background.

\section{Future Prospects}

The proposed future extension, IceCube-Gen2 detector [13], will have a factor of 5 to 10 times better sensitivity to astrophysical tau neutrinos than the current IceCube detector. With these large data samples and improvements in the reconstruction and background rejection techniques, described searches can lead to the detection of astrophysical tau neutrinos.

\section{References}

[1] M. G. Aartsen et al. (IceCube Collaboration), Science 342, 1242856 (2013).

[2] M. G. Aartsen et al. (IceCube Collaboration), Phys. Rev. Lett. 113, 101101 (2014).

[3] M. G. Aartsen et al. (IceCube Collaboration), Phys. Rev. Lett. 114, no. 17, 171102 (2015).

[4] M. G. Aartsen et al. (IceCube Collaboration), Astrophysical Journal 809, 98 (2015).

[5] F. Halzen, D. Hooper, Rept. Prog. Phys. 65, 1025 (2002).

[6] A. Levinson, E. Waxman, Phys. Rev. Lett. 87, 171101 (2001).

[7] R. Enberg, M. H. Reno, I. Sarcevic, Phys. Rev. D78, 043005 (2008).

[8] J. G. Learned, S. Pakvasa, Astroparticle Physics 3, 267 (1995).

[9] R. Abbasi et al. (IceCube Collaboration), Nucl. Instrum. Methods A 601, 294 (2009).

[10] M. G. Aartsen et al. (IceCube Collaboration), JINST 9, P03009 (2014).

[11] M. G. Aartsen et al. (IceCube Collaboration), arXiv: 1509.06212 [astro-ph.HE], (2015).

[12] D. Williams, M. Vraeghe, D. L. Xu, PoS (ICRC2015) 1071, (2015).

[13] M. G. Aartsen et al. (IceCube Collaboration), arXiv: 1412.5106 [astro-ph.HE], (2014). 